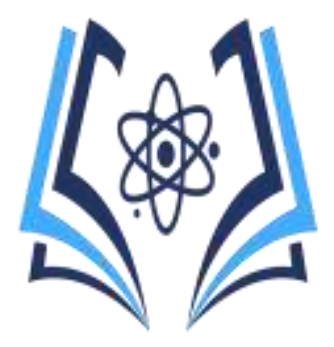

\title{
Cuidado integral de enfermería en personas con problemas de obesidad en la Parroquia Puerto Cayo.
}

\section{Comprehensive nursing care for people with obesity problems in Puerto Cayo Parish.}

\section{Cuidado integral de enfermería.}

\author{
María Josefina Espinel Menéndez ${ }^{1}$ \\ Chinga Bazurto Vicente Orlando ${ }^{2}$ \\ Delgado Carrillo Mercedes Jacqueline ${ }^{3}$
}

${ }^{1}$ Instituto Superior Universitario, Portoviejo, Ecuador. Email: majoespinel14@outlook.com
ORCID: https://orcid.org/0000-0003-1836-6265
${ }^{2}$ Instituto Superior Universitario, Portoviejo, Ecuador. Email: cvicente@itsup.edu.ec
ORCID: https://orcid.org/0000-0002-8819-6962
${ }^{3}$ Instituto Superior Universitario, Portoviejo, Ecuador. Email: dmercedes@itsup.edu.ec
ORCID: https://orcid.org/0000-0003-4636-4523

Contacto: majoespinel14@outlook.com

Recibido: 20-09-2020 Aprobado: 10-12-2020

\section{Resumen}

La obesidad es una enfermedad crónica que al igual que otras enfermedades si no recibe el tratamiento adecuado, en el tiempo puede llegar a complicar la salud de una persona, incluso le puede llevar a la muerte. El objetivo de esta investigación es determinar los cuidados de enfermería en personas que padecen este problema. La encuesta efectuada a 36 personas determinó que el $27,77 \%$ presentan obesidad y un $36,11 \%$ tienen problemas de sobrepeso, producto de malos hábitos en la alimentación, sumado a esto, otros factores como estilos de vida sedentarios, desconocimiento de las consecuencias que puede originarse a partir de esta patología, sin embargo, los encuestados están dispuestos a participar en los programas de cuidados que se propone en este proyecto. Por otra parte, se efectúo entrevistas a especialistas en medicina vinculados al tratamiento de problemas sobre la obesidad, en la cual los médicos desde diferentes enfoques de acuerdo a su especialidad ampliaron la información obtenida referente con respecto a este problema, en cuanto a detección, prevención y su tratamiento. En virtud de lo expuesto es muy importante que se continúe investigando, para prevenir y promocionar acciones enfocadas a sensibilizar a la población en general sobre la obesidad y sus consecuencias en la salud y por consiguiente en la calidad de vida.

Palabras claves: Obesidad, Sobrepeso, Malos hábitos, Alimentación, Estilos de vida

Summary

Obesity is a chronic disease that, like other diseases, if it does not receive adequate treatment, over time can complicate a person's health, it can even lead to death. The objective of this research is to determine the nursing care in people who suffer from this problem. The survey of 36 people determined that $27.77 \%$ have obesity and $36.11 \%$ have overweight problems, a product of bad eating habits, added to this, other factors such as sedentary lifestyles, ignorance of the consequences that can originate from this pathology, however, the 
respondents are willing to participate in the care programs proposed in this project. On the other hand, interviews were conducted with specialists in medicine linked to the treatment of obesity problems, in which doctors from different approaches according to their specialty expanded the information obtained regarding this problem, in terms of detection, prevention and its treatment. By virtue of the above, it is very important to continue research, to prevent and promote actions focused on sensitizing the general population about obesity and its consequences on health and consequently on quality of life.

Keywords: Obesity, Overweight, Bad habits, Diet, Lifestyles

\section{Introducción}

La obesidad se define como un acaparamiento anormal o excesiva de grasa que en grandes cantidades es perjudicial para la salud. La obesidad se calcula mediante el índice de masa corporal (IMC), que es una fórmula matemática simple, para esto se divide el peso de una persona en kilogramos para la estatura en metros elevada al cuadrado. Se considera a una persona obesa, cuando el IMC igual o superior a 30 (OMS, 2017).

La obesidad es una enfermedad crónica al igual que otras enfermedades que, si no recibe el tratamiento adecuado, en el tiempo puede llegar a complicar la salud de una persona, inclusive le puede llevar a la muerte. Dentro de las diferentes enfermedades que se pueden derivar de la obesidad tenemos malos hábitos en la alimentación, carencia de actividad física, predisposición genética, y en algunas ocasiones se generan en los ambientes de pobreza $\mathrm{y}$ entorno en el que se vive (surgery, 2020).

Diferentes estudios determinan que conforme la persona va adquiriendo mayor cantidad de grasa corporal, los riesgos de padecer o desarrollar una patología son cada vez mayores, es decir esta es una relación directamente proporcional. Entre estas patologías tenemos enfermedades coronarias, diabetes, hipertensión, cáncer, dislipidemia, accidentes cerebrovasculares, complicaciones ginecológicas, apnea, artrosis, patologías de hígado y vesícula, entre otras.

Cuando se diagnostica a un paciente con obesidad, inmediatamente se le debe abordar y explicar de forma sencilla y clara las consecuencias negativas para su salud que puede generar esta condición en la medida que siga aumentando esos niveles de peso. Por ello se hace imprescindible hacerle tomar conciencia para que cambie sus hábitos, sobre todo aquellos que son los factores que inciden directamente en esta situación.

Por otra parte, también genera en la persona afectada una serie de sensaciones en muchos casos desestabilizadoras en la parte emocional, pues lo primero que se pasa por sus pensamientos son sentimientos de depresión que pueden conllevar a una baja autoestima, por otra parte, también lo relacionan con la muerte, lo cual conlleva a una afectación no solo física, sino también en el ámbito social, laboral, económico y psicológico. También las personas del entorno que le rodean se ven afectadas, en mayor o menor grado, pues ello depende de muchos factores.

Según la Organización Mundial de la Salud (OMS) cada año mueren, alrededor de 2,8 millones de personas a causa de la obesidad en todo el mundo y de acuerdo a datos estadísticos hasta el 2016 más de 650 millones de personas padecían de este problema. La prevalencia de esta patología, se ha incrementado en el tiempo y desde 1975 hasta el 2016 se ha triplicado, siendo los países más desarrollados los que presentaban mayores índices de personas bajo esta condición de salud, sin embargo, durante las últimas décadas esta situación ya no es exclusiva de este segmento de la población, sino que afecta también a los países en vías de desarrollo y de pobreza en todos sus ámbitos (OMS, Organización Mundial de la Salud, 2016).

Para el 2016 alrededor de 41 millones de niños con edades menores a 5 años presentaban problemas de esta patología de acuerdo a datos proporcionados por la OMS, situación realmente preocupante pues incrementa las posibilidades de que años en el futuro desarrollen problemas de salud producto de esta situación, más aún cuando no se hace conciencia de la gravedad que esto puede generar sino no existe a tiempo el control adecuado de parte de los padres. Por otra parte, para el 2016, había entre niños y adolescentes 340 millones con problemas de obesidad y sobrepeso, cuyo aumento ha sido exponencial, ya que desde 1975 en el cual el $4 \%$ de este grupo presentaba dicho padecimiento, se elevó a más del 18\%, lo que representa aproximadamente 124 millones. Mientras que por encima del 39\% de la población mundial adulta al 2016, se veía afectada por esta situación. 
En el Ecuador 6 de cada 10 adultos padecen de obesidad, de acuerdo al Programa Mundial de Alimentos (PMA), para el 2030 se estima que la cantidad de muertos ocasionados por el sobrepeso y la obesidad se incrementarán a 13.000 personas más a las 22.671 que, en la actualidad, mueren cada año. De acuerdo a las estadísticas aumenta de manera exponencial en el Ecuador, y está presente en las 24 provincias de acuerdo al PMA, se destaca que solo la provincia de Napo presenta índices por debajo del $50 \%$, el resto de provincias sobrepasa ese porcentaje, siendo El Oro, Imbabura y el Guayas donde la prevalencia sobrepasa el $60 \%$. Lo que significa que 6 de cada 10 adultos en el Ecuador padecen este problema (Primicias, 2019).

El presente proyecto se lo realizó en Puerto Cayo, parroquia rural del cantón Jipijapa, perteneciente a la provincia de Manabí. Esta parroquia se encuentra ubicada en la costa suroeste del Ecuador en la conocida ruta del Spondylus que se extiende por el Océano Pacífico, está a una hora y 45 minutos desde Portoviejo; posee un clima agradable con una temperatura promedio de $24^{\circ} \mathrm{C}$, el cual en las noches llega a estar entre los $18^{\circ} \mathrm{C}$; además su clima se encuentra influenciado por la corriente fría de Humboldt, y la cálida del Niño, gracias a ello se establecen 2 épocas: el verano, que comprende los meses de Junio hasta Diciembre y por otra parte la época lluviosa, que empieza desde el mes de Enero hasta Mayo.

En lo que respecta a la parroquia Puerto Cayo, uno de los principales problemas de salud que afectan a esta población de acuerdo a información proporcionada por el centro de salud de este sector son enfermedades derivadas por la obesidad, existe un alto índice de personas que padecen esta patología la cual se ha venido incrementando de manera sustancial durante los últimos años.

El sedentarismo y los malos hábitos alimenticios estan dentro de los factores que establecen la prevalencia de este problema. Si bien es cierto el sector se caracteriza por la pesca de diversidad de especies marinas, y estas a su vez no solamente son una fuente de actividad para generar ingresos en las familias, sino que también hace parte de la dieta diaria de los habitantes y así ha sido por generaciones; sin embargo existen muchos factores externos que han modificado estos hábitos alimenticios, entre ellos mencionamos que existen muchos locales informales de venta de comida rápida como hamburguesas, salchipapas, pizas, entre otros, a esto le agregamos que los costos de estos productos son muy accesibles ya que con cincuenta centavos de dólar se puede adquirir algo de comer, con lo cual muchas familias optan por no preparar la merienda en casa y comer fuera..

La Atención Primaria de la enfermera incide por su gran accesibilidad al paciente y la longitudinalidad del tratamiento y seguimiento del mismo. Es imprescindible el dominio de conocimientos tales como son: alimentación equilibrada, los efectos de la dieta sobre la salud, la actividad física adecuada para cada paciente y establecer el compromiso por parte del paciente para ser persistente y disciplinado en el tratamiento.

El rol de la enfermería en este tipo de problemas es fundamental sobre todo en lo que respecta a la prevención, la cual involucra cuidados en la alimentación, para que esta sea una dieta con las calorías adecuadas de acuerdo a las necesidades del paciente. Descansar las horas adecuadas que requiere nuestro organismo para tener una funcionalidad óptima de nuestro cuerpo. Por otra parte, la actividad física es imprescindible porque no solamente ayuda a consumir las calorías que ingerimos, sino que una correcta planificación de esta actividad permite tener un peso equilibrado. Por otro lado, el rol de la enfermería también es trascendental en lo que respecta al cuidado de un paciente ya con problemas de salud originados por la obesidad. Aquí se requiere que el enfermero(a) siga junto al paciente de una manera disciplinada todas las recomendaciones a fin de lograr que de manera progresiva vaya disminuyendo los altos niveles de grasa corporal acumulados en su cuerpo, y a su vez esto conlleve a ir mejorando los problemas de salud.

La actividad física aquí también cumple un papel preponderante, los beneficios de realizar actividad física en pacientes con obesidad y sobrepeso serían: la mejora del perfil lipídico, la sensibilidad a la insulina y la presión arterial, la prevención de enfermedades cardiovasculares, el mantenimiento densidad ósea, la reducción del depósito de masa abdominal, el incremento de la capacidad respiratoria, entre otros. También la parte psicológica mejoraría a niveles positivos en lo referente a la autoestima, los problemas de ansiedad, depresión, etc (Madrid, 2020).

El objeto de estudio de la presente investigación es la obesidad, factor que está directamente vinculado o relacionado con la Bariatría, que es una rama de la medicina que se encarga de estudiar las causas, prevención y tratamiento en este caso de todo lo vinculado con la obesidad. 
El objetivo general de este proyecto es determinar los cuidados integrales de enfermería en personas con problemas de obesidad en la Parroquia Puerto Cayo, del cantón Jipijapa. De aquí se desprenden los siguientes objetivos específicos: en primera instancia se determinará los factores que generan

\section{Materiales y métodos}

Se realizó investigación de campo, para obtener información de primera mano y a la vez obtener datos precisos con respecto a los problemas que se identificaron en la Parroquia Puerto Cayo del cantón Jipijapa. Se utilizó el método inductivo y deductivo, ambos son estrategias de razonamiento lógico, siendo que el inductivo utiliza premisas particulares para llegar a una conclusión general, y el deductivo usa principios generales para llegar a una conclusión específica. Ambos métodos son importantes en la producción de conocimiento. Durante una investigación científica es posible que se utilice uno u otro, o una combinación de ambos, dependiendo del campo de estudio en el que se realice. Método Estadístico: El método estadístico los malos hábitos alimenticos, por otra parte, se va a identificar los daños que pueden ocasionar la obesidad en la salud de una persona; se establecerá una dieta equilibrada y además se recomendará actividad física diaria a los habitantes de esta parroquia.

consiste en una secuencia de procedimientos para el manejo de los datos cualitativos y cuantitativos de la investigación.

Para la realización de la presente investigación se aplicaron las siguientes técnicas:

Observación: Con el empleo de esta técnica se pudo conocer y adquirir información acerca de los habitantes de la Parroquia Puerto Cayo. Encuestas: Con esta técnica se obtuvo información de primera mano de los pobladores de esta parroquia con respecto al enfoque objetos de este estudio. Así mismo se realizó entrevistas a médicos y profesionales de la salud con la finalidad de obtener sus apreciaciones profesionales y poder contrastar con lo investigado.

\section{Resultados}

TABLA $\mathbf{N}^{\circ} 1$

Género de los encuestados

\begin{tabular}{|l|c|c|}
\hline \multicolumn{1}{|c|}{ ALTERNATIVAS } & F & \% \\
\hline Masculino & 22 & $61,10 \%$ \\
\hline Femenino & 14 & $38,90 \%$ \\
\hline TOTALES & $\mathbf{3 6}$ & $\mathbf{1 0 0 , 0 0 \%}$ \\
\hline
\end{tabular}

Los resultados nos indican que la mayoría de los encuestados son del género masculino, mientras que en menor cantidad pertenecen al género femenino.

TABLA $\mathbf{N}^{\circ} 2$

Edad de los encuestados

\begin{tabular}{|l|c|c|}
\hline \multicolumn{1}{|c|}{ ALTERNATIVAS } & F & \% \\
\hline Entre 18 y 30 años & & $33,33 \%$ \\
\hline Entre 31 y 40 años & 12 & $36,11 \%$ \\
\hline Entre 41 y 50 años & 13 & $22,22 \%$ \\
\hline Entre 51 y 60 años & 8 & $5,56 \%$ \\
\hline Entre 61 años en adelante & 2 & $2,78 \%$ \\
\hline TOTALES & 1 & $\mathbf{1 0 0 , 0 0 \%}$ \\
\hline
\end{tabular}


Las edades que prevalecen entre los encuestados, se encuentran en el rango comprendido entre 31 y 40 años de edad.

TABLA N $\mathbf{N}^{\circ} 3$

Peso corporal de los encuestados

\begin{tabular}{|l|c|c|}
\hline \multicolumn{1}{|c|}{ ALTERNATIVAS } & F & \% \\
\hline Entre $50 \mathrm{~kg}$ y $60 \mathrm{~kg}$ & 6 & $16,67 \%$ \\
\hline Entre $61 \mathrm{~kg}$ y $70 \mathrm{~kg}$ & 12 & $33,33 \%$ \\
\hline Entre $71 \mathrm{~kg}$ y $80 \mathrm{~kg}$ & 10 & $27,78 \%$ \\
\hline Entre $81 \mathrm{~kg}$ y $90 \mathrm{~kg}$ & 7 & $19,44 \%$ \\
\hline Entre $91 \mathrm{~kg}$ o más & 1 & $2,78 \%$ \\
\hline TOTALES & $\mathbf{3 6}$ & $\mathbf{1 0 0 , 0 0 \%}$ \\
\hline
\end{tabular}

Estos resultados nos permiten evidenciar que la mayoría de encuestados, tienen un peso promedio establecido en el rango de 61 a 70 kilogramos.

TABLA No 4

Estatura de los encuestados

\begin{tabular}{|l|c|c|}
\hline \multicolumn{1}{|c|}{ ALTERNATIVAS } & F & \% \\
\hline Entre $1,40 \mathrm{~m}$ y $1,50 \mathrm{~m}$ & 2 & $5,56 \%$ \\
\hline Entre $1,51 \mathrm{~m}$ y $1,60 \mathrm{~m}$ & 16 & $44,44 \%$ \\
\hline Entre $1,61 \mathrm{~m}$ y $1,70 \mathrm{~m}$ & 15 & $41,67 \%$ \\
\hline Más de $1,71 \mathrm{~m}$ & 3 & $8,33 \%$ \\
\hline TOTALES & $\mathbf{3 6}$ & $\mathbf{1 0 0 . 0 0 \%}$ \\
\hline
\end{tabular}

La estatura promedio que prevalece entre las personas encuestadas se encuentra en el rango de 1,51 metros a 1,60 metros.

TABLA $\mathbf{N}^{\circ} 5$

Índice de masa corporal (IMC)

\begin{tabular}{|l|c|c|}
\hline \multicolumn{1}{|c|}{ ALTERNATIVAS } & F & \% \\
\hline$<18,49$ (Delgadez) & 2 & $5.56 \%$ \\
\hline Entre 18,50 y 24,99 (Normal) & 8 & $22.22 \%$ \\
\hline Entre 25 y 29,99 (Sobrepeso) & 13 & $36.11 \%$ \\
\hline Más de 30 (Obeso) & 13 & $36.11 \%$ \\
\hline TOTALES & $\mathbf{3 6}$ & $\mathbf{1 0 0 . 0 0 \%}$ \\
\hline
\end{tabular}

Los datos obtenidos permiten establecer que existen problemas de sobrepeso y obesidad en la mayoría de los encuestados, con lo cual el presente proyecto de investigación se fortalece en su propósito inicialmente establecido. En entrevista realizada a la Dra. Liliana Ponce, manifestó que se considera a una persona con obesidad cuando la valoración obtenida al realizar el cálculo del IMC es igual o superior a 30 . 
TABLA $\mathbf{N}^{\circ} 6$

Frecuencia de consumo de alcohol

\begin{tabular}{|l|c|c|}
\hline \multicolumn{1}{|c|}{ ALTERNATIVAS } & F & \% \\
\hline Frecuentemente & 8 & $22,22 \%$ \\
\hline Rara vez & 21 & $58,33 \%$ \\
\hline Nunca & 7 & $19,45 \%$ \\
\hline TOTALES & $\mathbf{3 6}$ & $\mathbf{1 0 0 , 0 0 \%}$ \\
\hline
\end{tabular}

Con respecto al consumo de alcohol, los resultados de la encuesta determinaron que los encuestados rara vez lo hacen, lo que evidencia que este no es uno de los factores principalmente asociado que propicia a que las personas puedan padecer de problemas de peso corporal.

TABLA N ${ }^{\circ} 7$

Factores que inciden para que las personas consuman alimentos preparados fuera de casa

\begin{tabular}{|l|c|c|}
\hline \multicolumn{1}{|c|}{ ALTERNATIVAS } & F & \% \\
\hline Por el trabajo & 15 & $41,66 \%$ \\
\hline Por no cocinar & 15 & $41,66 \%$ \\
\hline Porque en casa no hay quien prepare los alimentos & 4 & $11,11 \%$ \\
\hline Porque la comida en la calle es más barata & 2 & $5,57 \%$ \\
\hline Porque la comida en la calle es mejor & 0 & $0,00 \%$ \\
\hline Otra & 0 & $0,00 \%$ \\
\hline TOTALES & $\mathbf{3 6}$ & $\mathbf{1 0 0 , 0 0 \%}$ \\
\hline
\end{tabular}

Con respecto a los factores que inciden para que las personas consuman alimentos fuera de casa los resultados permiten establecer que los dos factores que prevalecen son el trabajo y por no preparar los alimentos en casa.

TABLA $\mathbf{N}^{\circ} 8$

Productos que consumen con mayor frecuencia.

\begin{tabular}{|l|c|c|}
\hline \multicolumn{1}{|c|}{ ALTERNATIVAS } & F & \% \\
\hline Bebidas gaseosas & 27 & $32,92 \%$ \\
\hline Jugos envasados & 13 & $15,85 \%$ \\
\hline Hamburguesas & 15 & $18,29 \%$ \\
\hline Snaks & 5 & $6,09 \%$ \\
\hline Hot Dog & 3 & $3,66 \%$ \\
\hline Pizza & 3 & $3,66 \%$ \\
\hline Frituras envasadas & 10 & $12,19 \%$ \\
\hline Ninguna & 6 & $7,34 \%$ \\
\hline TOTALES & $\mathbf{8 2}$ & $\mathbf{1 0 0 , 0 0 \%}$ \\
\hline
\end{tabular}

Con respecto a los productos que consumen con mayor frecuencia, los resultados indican que prevalece la ingesta de bebidas gaseosas, lo cual se constituye en unos de los principales factores que van asociados a los problemas de sobrepeso y obesidad detectados. 
TABLA $\mathbf{N}^{\circ} 9$

Frecuencia de consumo de alimentos fuera del horario de las comidas habituales.

\begin{tabular}{|l|c|c|}
\hline \multicolumn{1}{|c|}{ ALTERNATIVAS } & F & \% \\
\hline A cualquier hora del día & 18 & $50,00 \%$ \\
\hline En la noche antes de acostarse & 10 & $27,78 \%$ \\
\hline Sólo consume las tres comidas diarias & 8 & $22,22 \%$ \\
\hline TOTALES & $\mathbf{3 6}$ & $\mathbf{1 0 0 , 0 0 \%}$ \\
\hline
\end{tabular}

Con respecto al consumo de alimentos fuera del horario habitual de comidas, la respuesta que prevaleció con mayor frecuencia fue la ingesta de alimentos a cualquier hora del día.

TABLA $\mathbf{N}^{\circ} 10$

Número de familiares con sobrepeso u obesidad

\begin{tabular}{|l|c|c|}
\hline \multicolumn{1}{|c|}{ ALTERNATIVAS } & F & \% \\
\hline Ninguna & 9 & $25,00 \%$ \\
\hline Entre 1 y 2 familiares & 20 & $55,56 \%$ \\
\hline Entre 3 y 4 familiares & 5 & $13,89 \%$ \\
\hline Más de 5 familiares & 2 & $5,55 \%$ \\
\hline TOTALES & $\mathbf{3 6}$ & $\mathbf{1 0 0 , 0 0 \%}$ \\
\hline
\end{tabular}

Se consultó para conocer cuántos miembros de la familia padecían de sobrepeso u obesidad, y los resultados permitieron determinar que en la mayoría de los encuestados tienen uno o dos familiares con problemas de sobrepeso u obesidad.

TABLA $\mathbf{N}^{\circ} 11$

Familiares con antecedentes de diabetes

\begin{tabular}{|l|c|c|}
\hline \multicolumn{1}{|c|}{ ALTERNATIVAS } & F & \% \\
\hline Padre & 4 & $11,11 \%$ \\
\hline Madre & 5 & $13,89 \%$ \\
\hline Abuelos & 14 & $38,89 \%$ \\
\hline Ninguno & 13 & $36,11 \%$ \\
\hline TOTALES & $\mathbf{3 6}$ & $\mathbf{1 0 0 , 0 0 \%}$ \\
\hline
\end{tabular}

Se indagó si algún miembro de su familia tiene diabetes, los resultados indican que los abuelos maternos o paternos padecen esta enfermedad, lo cual constituye como un factor de riesgo, considerando que esta enfermedad puede ser genéticamente hereditaria. De acuerdo a lo expresado por el Dr. Víctor Rodríguez señaló que una de las patologías que puede originar o desencadenar la obesidad es la diabetes. 
TABLA $\mathrm{N}^{\circ} 12$

Familiares con antecedentes de hipertensión

\begin{tabular}{|l|c|c|}
\hline \multicolumn{1}{|c|}{ ALTERNATIVAS } & F & \% \\
\hline Padre & 2 & $5,55 \%$ \\
\hline Madre & 9 & $25,00 \%$ \\
\hline Abuelos & 8 & $22,22 \%$ \\
\hline Hijos & 0 & $0,00 \%$ \\
\hline Ninguno & 17 & $47,23 \%$ \\
\hline TOTALES & $\mathbf{3 6}$ & $\mathbf{1 0 0 , 0 0 \%}$ \\
\hline
\end{tabular}

Al consultar sobre familiares con problemas de hipertensión, la mayoría de los encuestados

manifestó no tener ningún familiar con esta patología.

TABLA $\mathrm{N}^{\circ} 13$

Frecuencia con la que se realizan exámenes clínicos

\begin{tabular}{|l|c|c|}
\hline \multicolumn{1}{|c|}{ ALTERNATIVAS } & F & \% \\
\hline Cada 6 meses & 3 & $8,33 \%$ \\
\hline Cada año & 3 & $8,33 \%$ \\
\hline Cuando se presenta una enfermedad & 30 & $83,34 \%$ \\
\hline TOTALES & $\mathbf{3 6}$ & $\mathbf{1 0 0 , 0 0 \%}$ \\
\hline
\end{tabular}

La frecuencia con qué se realizan exámenes clínicos para conocer el estado de salud personal y de los miembros de la familia permiten establecer que no existe una cultura de prevención en la salud, debido a que la mayoría de personas no se realizan exámenes clínicos de prevención, sino cuando se presenta una enfermedad.

\section{TABLA $\mathrm{N}^{\circ} 14$}

Frecuencia con la que realiza actividad física

\begin{tabular}{|l|c|c|}
\hline \multicolumn{1}{|c|}{ ALTERNATIVAS } & F & $\mathbf{\%}$ \\
\hline 1 vez por semana & 11 & $30,56 \%$ \\
\hline 3 veces por semana & 7 & $19,44 \%$ \\
\hline 5 veces por semana & 1 & $2,78 \%$ \\
\hline Todos los días & 0 & $0,00 \%$ \\
\hline No realiza ninguna actividad física & 17 & $47,22 \%$ \\
\hline TOTALES & $\mathbf{3 6}$ & $\mathbf{1 0 0 , 0 0 \%}$ \\
\hline
\end{tabular}

La frecuencia con la que los encuestados realizan actividad física, prevaleció en su mayoría que no realizan ningún tipo de actividad física.

TABLA $\mathrm{N}^{\circ} 15$

Factor que influye para que no realice actividad física

\begin{tabular}{|l|c|c|}
\hline \multicolumn{1}{|c|}{ ALTERNATIVAS } & F & \% \\
\hline El trabajo & 19 & $47,50 \%$ \\
\hline Por problemas de salud & 0 & $0,00 \%$ \\
\hline Por los estudios & 5 & $12,50 \%$ \\
\hline
\end{tabular}

https://revistas.itsup.edu.ec/index.php/higia 


\begin{tabular}{|l|c|c|} 
Por falta de motivación & 12 & $30,00 \%$ \\
\hline No lo considera importante & 3 & $7,50 \%$ \\
\hline Otra & 1 & $2,50 \%$ \\
\hline TOTALES & $\mathbf{4 0}$ & $\mathbf{1 0 0 , 0 0 \%}$ \\
\hline
\end{tabular}

Entre los factores que impiden que se realice actividad física con frecuencia los resultados obtenidos, indicaron que el factor que les impide llevar una rutina de ejercicios durante la semana es el trabajo.

TABLA $\mathrm{N}^{\circ} 16$

Campaña sobre promoción de buenos hábitos alimenticios y estilo de vida.

\begin{tabular}{|l|c|c|}
\hline \multicolumn{1}{|c|}{ ALTERNATIVAS } & F & \% \\
\hline Totalmente de acuerdo & 35 & $97,22 \%$ \\
\hline No estoy de acuerdo & 0 & $0,00 \%$ \\
\hline Le es indiferente & 1 & $2,78 \%$ \\
\hline TOTALES & $\mathbf{3 6}$ & $\mathbf{0 , 0 0 \%}$ \\
\hline
\end{tabular}

Al investigar sí estaría de acuerdo con que se realice una campaña para mejorar los hábitos alimenticios y estilo de vida, los encuestados expresaron estar totalmente de acuerdo en su gran mayoría, lo que representa la apertura de los habitantes para campañas de promoción de la salud sobre la obesidad.

TABLA No 17

Visitas a los hogares para campaña de promoción.

\begin{tabular}{|l|c|c|}
\hline \multicolumn{1}{|c|}{ ALTERNATIVAS } & F & \% \\
\hline Si lo considero importante & 31 & $86,11 \%$ \\
\hline No lo considero importante & 2 & $5,56 \%$ \\
\hline Le es indiferente & 3 & $8,33 \%$ \\
\hline TOTALES & $\mathbf{3 6}$ & $\mathbf{1 0 0 , 0 0 \%}$ \\
\hline
\end{tabular}

Al consultar si consideraban importante que se realice visitas coordinadas en el hogar conformada por un equipo de profesionales de la salud con la finalidad de diagnosticar y hacer un seguimiento de los casos de alto IMC detectados, los resultados establecieron que los encuestados están predispuestos y lo consideran importante.

\section{Discusión}

La investigación se desarrolla en función del alto índice de problemas de obesidad en los habitantes de la Parroquia Puerto Cayo debido a los malos hábitos alimenticios y a la vida sedentaria que llevan en su mayoría, sin darse cuenta que este problema va aumentando sobre todo en los niños que desde temprana edad ya presentan un índice de masa corporal (IMC) más alto del promedio normal de acuerdo a las edades.

La obesidad es un padecimiento ligado a múltiples factores que inciden de manera negativa en la salud de una persona, radica en tener de forma excesiva gran cantidad de grasa corporal. La obesidad como tal, no es solo un problema de carácter estético, sino que también es un problema que desarrolla riesgos de enfermedades y complicaciones en la salud, entre ellos enfermedades cardíacas, diabetes, problemas de presión arterial y en muchos casos se ha detectado algunas tipologías de cáncer

El objetivo de este proyecto es determinar los cuidados integrales de enfermería en personas con problemas de obesidad en la Parroquia Puerto Cayo, del cantón Jipijapa, con la finalidad de atender a grupos vulnerables creando una cultura de buenos hábitos alimenticios y la práctica permanente de actividad física, conllevando todo esto a reducir los índices de obesidad a corto y mediano plazo, con lo cual esto derivará en personas con una buena salud física y mental. 
El rol de la enfermería en este tipo de problemas es fundamental sobre todo en lo que respecta a la prevención, la cual involucra cuidados en la alimentación, para que esta sea una dieta con las calorías adecuadas de acuerdo a las necesidades del paciente. Descansar las horas adecuadas que requiere nuestro organismo para tener una funcionalidad óptima de nuestro cuerpo. Por otra parte, la actividad física es imprescindible porque no solamente ayuda a consumir las calorías que ingerimos, sino que una correcta planificación de esta actividad permite tener un peso equilibrado.

Por otro lado, el rol de la enfermería también es trascendental en lo que respecta al cuidado de un paciente ya con problemas de salud originados por la obesidad. Aquí se requiere que el enfermero(a) siga junto al paciente de una manera disciplinada todas las recomendaciones a fin de lograr que de manera progresiva vaya disminuyendo los altos niveles de grasa corporal acumulados en su cuerpo, y a su vez esto conlleve a ir mejorando los problemas de salud.

Durante mucho tiempo la obesidad fue considerada como un trastorno con fuertes determinantes en la conducta de los niños y adolescentes manifestado psicopatológicamente en la alimentación, según investigaciones realizadas se conoce la causa por la cual se estipulan múltiples factores cada uno en distintas dimensiones afectando a todos por igual sin importar sexo, talla, edad (Pallo García, 2017).

Por otra parte, se coincide en criterios en la que se hace énfasis en que la alimentación y el sedentarismo en los escolares con sobrepeso y obesidad son los principales factores de riesgo que afectan la salud de este grupo poblacional siendo la diabetes y la hipertensión complicaciones que surgen en la edad adulta cuando no son controladas adecuadamente (Mezones Acebo, 2019).
A través de la investigación se propuso lograr un nivel de sensibilización en los pobladores de la Parroquia Puerto Cayo con respecto a los problemas que se pueden desencadenar con el sobrepeso y la obesidad. Se logró establecer cuáles son los factores asociados a los problemas de peso corporal, así como también las acciones que las autoridades correspondientes han realizado con respecto a esta problemática. Al implementar programas de medicina preventiva y también de tratamientos orientados a personas con problemas de obesidad bajo el consentimiento y aprobación de los habitantes de esta Parroquia, indudablemente que constituye una respuesta efectiva y positiva con respecto a este problema.

\section{Conclusiones}

Esta investigación permitió conocer los problemas de sobre peso y obesidad por los que padece un gran número de habitantes de la Parroquia Puerto Cayo del cantón Jipijapa, deja muy claro que los malos hábitos alimenticios e inadecuados estilos de vida, como el sedentarismo son los principales factores asociados a esta problemática. Por otra parte, es muy importante resaltar el nivel de sensibilidad y conciencia que existe entre las familias con respecto a esto y la predisposición para ser parte de campañas enfocadas en la prevención y en los casos donde ya existe el problema los diferentes tipos de tratamientos.

La implementación de cuidados integrales de enfermería para los habitantes de la Parroquia Puerto Cayo se constituye en un programa pionero, que servirá como base para estudios futuros con respecto a esta y otras problemáticas de salud que están presentes. Estas estrategias están orientadas a crear buenos hábitos en la alimentación y formas de vida y con ello se bajarán los índices de problemas de salud derivados por el sobrepeso $\mathrm{u}$ obesidad.

\section{Bibliografía}

Madrid, C. M. (22 de marzo de 2020). Centro Médico y de Rehabilitación Premium Madrid. Obtenido de Centro Médico y de Rehabilitación Premium Madrid: https://rehabilitacionpremiummadrid.com/blog/sara-carnicero/la-importancia-de-la-actividadfisica-en-la-prevencion-y-el-tratamiento-de-la-obesidad-y-sobrepeso-en-adultos-partei/\#: :text=Adem\%C3\%A1s\%20de\%20lo\%20comentado\%20hasta,arterial\%2C\%20la\%20prevenc

OMS. (14 de noviembre de 2016). Organización Mundial de la Salud. Obtenido de Organización Mundial de la Salud: https://www.who.int/features/factfiles/obesity/es/

OMS. (10 de marzo de 2017). Organización Mundial de la Salud. Obtenido de Organización Mundial de la Salud: https://www.who.int/features/factfiles/obesity/es/ 
Primicias, D. D. (20 de mayo de 2019). Diario Digital Primicias. Obtenido de Diario Digital Primicias: https://www.primicias.ec/noticias/sociedad/sobrepeso-obesidad-muerte-alimentos/

surgery, I. f. (6 de marzo de 2020). Innovation for surgery. Obtenido de Innovation for surgery: https://www.mid-med.com/es/surgery/comprendiendo-la-obesidad/ 